\title{
Effect of early mobilization and rehabilitation on complications in aneurysmal subarachnoid hemorrhage
}

\author{
Tanja Karic, MD,1,2 Cecilie Røe, MD, PhD, ${ }^{1,4}$ Tonje Haug Nordenmark, PhD, ${ }^{1}$ \\ Frank Becker, MD, PhD, ${ }^{3,4}$ Wilhelm Sorteberg, MD, PhD, ${ }^{2}$ and Angelika Sorteberg, MD, $\mathrm{PhD}^{2,4}$ \\ Departments of ${ }^{1}$ Physical Medicine and Rehabilitation and ${ }^{2}$ Neurosurgery, Oslo University Hospital, Oslo; \\ ${ }^{3}$ Sunnaas Rehabilitation Hospital, Nesoddtangen; and ${ }^{4}$ Institute of Clinical Medicine, University of Oslo, Norway
}

\begin{abstract}
OBJECTIVE Early rehabilitation is effective in an array of acute neurological disorders but it is not established as part of treatment guidelines after aneurysmal subarachnoid hemorrhage (aSAH). This may in part be due to the fear of aggravating the development of cerebral vasospasm, which is the most feared complication of aSAH. The aim of this study was to evaluate the effect of early rehabilitation and mobilization on complications during the acute phase and within 90 days after aSAH.
\end{abstract}

METHODS This was a prospective, interventional study that included patients with aSAH at the neuro-intermediate ward after aneurysm repair. The control group received standard treatment, whereas the early rehab group underwent early rehabilitation and mobilization in addition to standard treatment. Clinical and radiological characteristics of patients with aSAH, progression in mobilization, and treatment variables were registered. The frequency and severity of cerebral vasospasm, cerebral infarction acquired in conjunction with the aSAH, and acute and chronic hydrocephalus, as well as pulmonary and thromboembolic complications, were compared between the 2 groups.

RESULTS Clinical and radiological characteristics of patients with aSAH were similar between the groups. The early rehab group was mobilized beginning on the first day after aneurysm repair. The significantly quicker and higher degree of mobilization in the early rehab group did not increase complications. Clinical cerebral vasospasm was not as frequent in the early rehab group and it also tended to be less severe. Each step of mobilization achieved during the first 4 days after aneurysm repair reduced the risk of severe vasospasm by $30 \%$. Acute and chronic hydrocephalus were similar in both groups, but there was a tendency toward earlier shunt implantation among patients in the control group. Pulmonary infections, thromboembolic events, and death before discharge or within 90 days after the ictus were similar between the 2 groups.

CONCLUSIONS Early rehabilitation of patients after aSAH is safe and feasible. The earlier and higher degree of mobilization does not increase neurosurgical complications. Rather, the frequency and severity of cerebral vasospasm following aSAH are alleviated and are not aggravated by early rehabilitation.

Clinical trial registration no.: NCT01656317 (www.clinicaltrials.gov).

http://thejns.org/doi/abs/10.3171/2015.12.JNS151744

KEY WORDS aneurysmal subarachnoid hemorrhage; cerebral vasospasm; complications; early rehabilitation; mobilization; neurointensive care; vascular disorders

A NEURYSMAL subarachnoid hemorrhage (aSAH) is a life-threatening event. Apart from the deleterious effects of the ictus per se, patients who suffer aSAH are at risk for developing a wide range of complications secondary to their hemorrhage..$^{41}$ These complications are important because they may result in worse outcome. Therefore, consecutive to early aneurysm repair, intensive care management of patients with aSAH focuses on the prevention of additional brain damage. Delayed cerebral ischemic events are particularly feared and represent a crucial risk factor for poor neurological outcome and increased case fatality. ${ }^{2}$

Intensive care management of patients with aSAH could last for weeks. Prolonged bed rest and immobility may then increase the physical impairments ${ }^{28}$ that early rehabilitation interventions may alleviate or prevent. ${ }^{28,38}$

ABBREVIATIONS aSAH = aneurysmal subarachnoid hemorrhage; $\mathrm{CPP}=$ cerebral perfusion pressure; $\mathrm{CTA}=\mathrm{CT}$ angiography; $\mathrm{DVT}=$ deep venous thrombosis; NIW = neuro-intermediate ward; $P E=$ pulmonary embolism.

SUBMITTED July 26, 2015. ACCEPTED December 29, 2015.

INCLUDE WHEN CITING Published online April 8, 2016; DOI: 10.3171/2015.12.JNS151744. 
Although it is generally accepted that early rehabilitation is effective in an array of acute neurological disorders, $3,8-11,13,18,28,35$ it has not been established as part of treatment guidelines for patients after aSAH. ${ }^{37} \mathrm{~A}$ main barrier to mobilization has been the fear of adverse effects such as the development of cerebral vasospasm and the increased risk of secondary brain damage.

Although body positioning and mobilization are crucial elements of early rehabilitation, there is no general consensus regarding these topics in patients with aSAH. ${ }^{6,37}$ Positioning regimens are largely based on traditions and beliefs at individual institutions and not primarily on welldocumented physiological responses. ${ }^{7,28}$ Olkowski et al. ${ }^{31}$ concluded that early rehabilitation was safe and feasible in patients with aSAH, but they did not address in detail the occurrence of secondary complications, especially the most feared secondary complication of aSAH: cerebral vasospasm.

It may be assumed that there would be a subtle balance between rehabilitation efforts aimed at preventing the consequences of prolonged bed rest on the one hand, and treatment efforts aimed at preventing secondary brain injury on the other hand. The goal of the present study was to evaluate the effect of early mobilization and rehabilitation on complications during the acute phase after aSAH.

\section{Methods}

\section{Patient Characteristics}

This prospective, controlled interventional study included all patients who were managed at the neuro-intermediate ward (NIW) of the Neurosurgical Department at Oslo University Hospital, Rikshospitalet (Oslo, Norway), following repair of a ruptured intracranial aneurysm during the time period 2011-2012.

Patients admitted during 2011 received standard treatment in accordance with our institutional guidelines ${ }^{36}$ and they constituted the control group. Patients admitted during 2012 represented the intervention group that underwent early mobilization and rehabilitation in addition to standard treatment, thereafter denoted the early rehab group. Exclusion criteria were age under 18 years and a history of SAH, traumatic brain injury, or neurodegenerative disorder.

The present study is part of a larger project entitled "The effect of early rehabilitation after aneurysmal SAH." The study adheres to the principles set forth in the US Code of Federal Regulations, Title 45, Part 46, Protection of Human Subjects, and the World Medical Association Declaration of Helsinki. The project was approved by the Regional Committee for Medical Research Ethics, Southeast Norway, in January 2012, archive number 2011/2189, clinical trial number 0925-0586 (www.ClinicalTrials. gov; NCT01656317). Oral and written informed consent was obtained from all patients included in the study.

\section{Methods and Criteria}

\section{Standard Institutional aSAH Management Principles}

Because it is part of a tertiary hospital with a catchment area of 2.7 million people, our neurosurgical department manages 120-150 patients with aSAH each year. Surgical and endovascular aneurysm repair is available 24 hours every day and is performed by dedicated vascular teams. Patients are treated in accordance with standardized guidelines, ${ }^{36}$ including ultra-early aneurysm repair, liberal CSF drainage, maintenance of cerebral perfusion pressure (CPP) $>70 \mathrm{~mm} \mathrm{Hg}$, and use of intravenous or oral nimodipine for 3 weeks after the ictus. While on invasive mechanical ventilatory support, patients are managed at the general ICU under neurosurgical supervision; otherwise, patients are managed at the NIW, where the nursing staff is specially trained in the care of patients with aSAH. When they no longer need neurosurgical treatment, patients are transferred to their local ICUs or neurological/ rehabilitation departments.

We conducted cerebral CT angiography (CTA) before aneurysm repair, the day after aneurysm repair, and 7 days after the ictus. We also performed cerebral CTA and transcranial Doppler ultrasonography if vasospasm was suspected clinically. Transcranial Doppler ultrasonography was often used to tailor patient management for those with vasospasm. When severe cerebral vasospasm was diagnosed, the CPP threshold was elevated to $>90 \mathrm{~mm} \mathrm{Hg}$ by means of volume expansion and lowering of the intracranial pressure toward $0 \mathrm{~mm} \mathrm{Hg}$ through aggressive CSF drainage, and by pharmacological increase of the mean arterial blood pressure. If these measures were deemed insufficient, patients were treated with intraarterial injection of nimodipine.

\section{Intervention: Early Mobilization and Rehabilitation}

Early mobilization was conducted according to a stepwise mobilization algorithm,,$^{20}$ beginning with the first day after aneurysm repair (Table 1). A goniometer was used to determine the degree of head elevation. Step progression in mobilization depended on individually defined thresholds of surveillance parameters such as mean arterial blood pressure, intracranial pressure, CPP, oxygenation, carbon dioxide partial pressure, heart rate, respiratory frequency, lack of neurological deterioration, and no reported patient discomfort. From Steps 0 to 2, there was a minimum of 1 day for each step in the progression; progression from Steps 2 to 6 could be quicker. Moderate vasospasm did not delay mobilization. In the event of severe and/or clinical vasospasm, mobilization was halted or reversed to Step 0 .

Early rehabilitation was based on an interdisciplinary approach $^{19}$ and was initiated upon the patient's arrival to the NIW after aneurysm repair. The content of early rehabilitation was as follows: neutral positioning, passive

\section{TABLE 1. Mobilization algorithm}

\begin{tabular}{cl}
\hline Step & \multicolumn{1}{c}{ Level of Mobilization } \\
\hline 0 & Bed rest, head elevation $30^{\circ}$ \\
\hline 1 & Bed rest, head elevation $60^{\circ}$ \\
\hline 2 & Bed rest, head elevation $80^{\circ}$ \\
\hline 3 & Sitting on the edge of the bed \\
\hline 4 & Sitting in a chair \\
\hline 6 & Standing at bedside, walking a few steps at bedside \\
\hline
\end{tabular}


joint movements, pulmonary rehabilitation, guidance in activities of daily living, reality orientation, and information and emotional support to patients and their families. ${ }^{20}$

\section{Variables Registered}

We registered patient demographic data and SAH characteristics along with treatment variables and the following complications: cerebral vasospasm, new radiologically verified cerebral infarction, hydrocephalus, and respiratory tract/pulmonary infection. Deep venous thrombosis (DVT), pulmonary embolism (PE), and death within 90 days were also registered.

Clinical cerebral vasospasm was defined as "delayed neurological deterioration that could not be attributed to rebleeding, hydrocephalus, intracerebral hematoma, electrolyte abnormalities, or toxic and metabolic factors." ${ }^{5}$

Respiratory tract/pulmonary infection was diagnosed if the patient had a body temperature $\geq 38^{\circ} \mathrm{C}$ combined with elevated blood sample infection parameters and at least 1 of the following: new onset of purulent sputum, increased oxygen requirements, radiological evidence, or increased ventilatory demand. Deep venous thrombosis was diagnosed by clinical evidence confirmed by ultrasound or venography of the extremities, whereas PE was diagnosed by clinical evidence confirmed by thoracic CTA.

\section{Statistical Analysis}

Analysis was performed using SPSS version 22 (SPSS,
Inc.). Continuous variables that were normally distributed are presented as the mean and standard deviation (SD), and the independent samples t-test was used to compare differences between groups. Continuous variables that were not normally distributed are presented as the median and range, and the Mann-Whitney U-test was used to compare differences between groups. Categorical variables are presented as frequencies or percentages and the chi-square test was used to compare differences between groups. We used univariate and multivariate regression analysis to identify predictors of severe and clinical vasospasm. A significance level of 0.05 was used.

For sample size calculations, we assumed effects of early rehabilitation similar to those observed by Andelic et al. ${ }^{3}$ Thus, 25 patients would be necessary in each group, given $90 \%$ power and a significance level of 0.05 . With 120 aSAH cases per year, and $60 \%$ of them meeting the inclusion criteria with a $15 \%$ dropout rate, at least twice the number of patients necessary should accrue within the 1 -year inclusion period.

\section{Results}

\section{Patients Included in Control and Early Rehab Groups}

Figure 1 shows a flow chart of eligible patients included in the study. A total of 77 patients were included in the control group and 94 patients were included in the early rehab group.

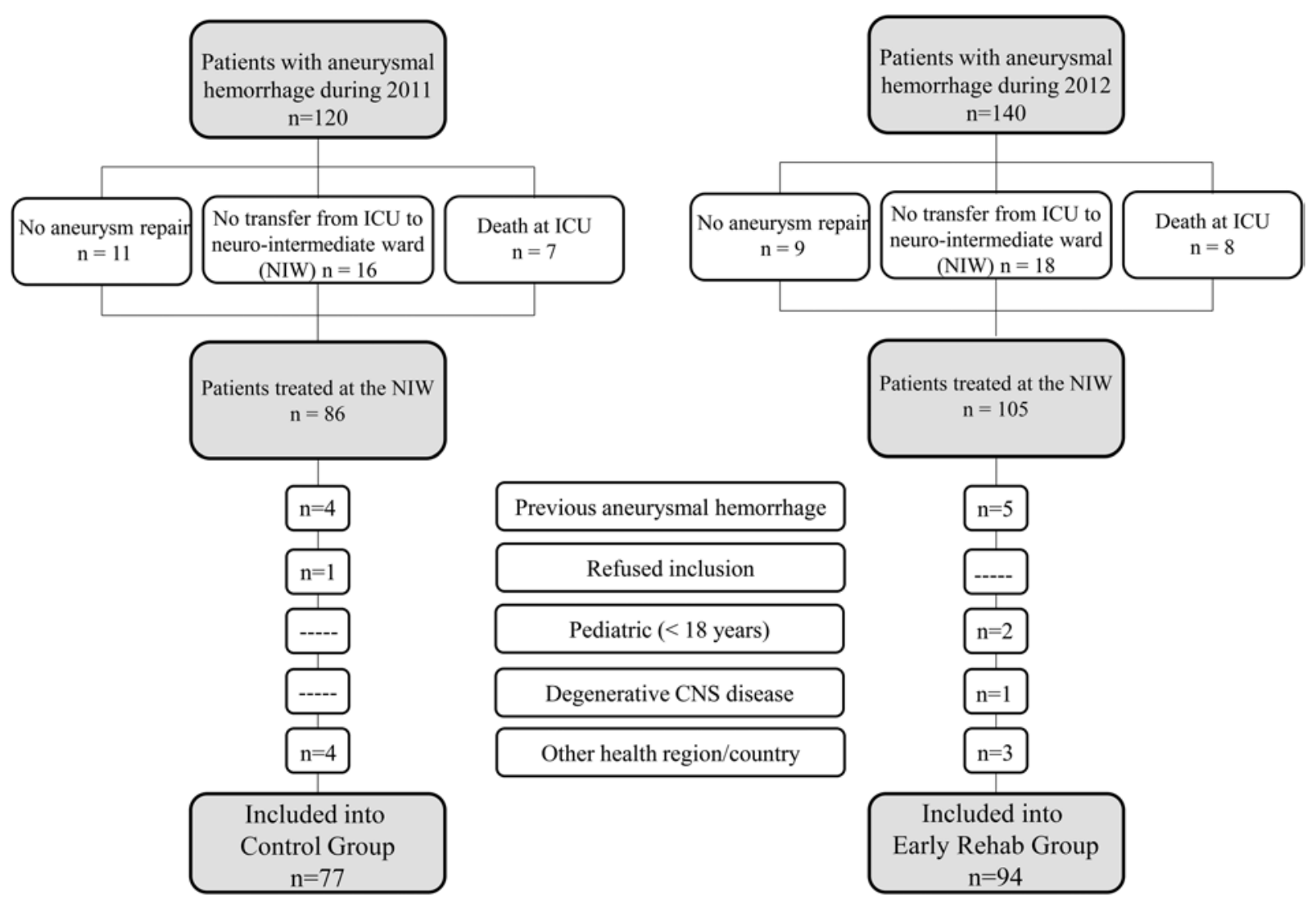

FIG. 1. Flow chart of eligible patients included in the study. 


\section{Entrance Variables}

The entrance variables for both groups are presented in Table 2 . There were no statistically significant differences between the control group and the early rehab group.

\section{Treatment Variables}

Table 3 presents treatment variables in the control group and in the early rehab group. Except for significantly more patients in the control group receiving corticosteroids, no treatment variable differed significantly between the groups. There was a trend toward more use of heparin after aneurysm repair $(\mathrm{p}=0.061)$ and a tendency toward earlier shunt placement in the control group (24 vs 32 days; $p=0.07$ ).

\section{Intervention: Early Mobilization and Rehabilitation}

The median mobilization step upon arrival to the NIW was 0 (bed rest, head elevation $30^{\circ}$ ) in both patient groups. Patients in the early rehab group were mobilized significantly earlier than those in the control group (Fig. 2; p < 0.01 for Days 1-7). Likewise, patients in the early rehab group had reached a higher mobilization level at discharge $(\mathrm{p}=0.004)$ than those in the control group. At discharge (Fig. 3), patients in the early rehab group were hence on average at mobilization Step 5 (walking a few steps at bedside), whereas patients in the control group were on average at Step 4 (sitting in a chair). None of the patients suffered an accidental fall during early mobilization nor had episodes of unintended removal of tubes/lines during early rehabilitation.

\section{Cerebral Vasospasm}

Fifteen patients (5 in the control group and 10 in the early rehab group) were excluded from this analysis because they had bled some days prior to hospitalization and had developed cerebral vasospasm upon arrival to our hospital.

The time from ictus to vasospasm was not significantly different between the 2 groups (control group: median 7 days [range 4-22 days]; early rehab group: median 8 days [range 3-18 days]). The frequencies of radiological, ultrasonic, and clinical vasospasm in the 2 groups are presented in Table 4. There was significantly less clinical vasospasm in the early rehab group $(p=0.03)$. Moreover, there was a tendency toward less frequent radiological and ultrasonic vasospasm in the early rehab group. Comparison of mobilization step with degree of cerebral vasospasm revealed a trend toward less vasospasm in the early rehab group (Fig. 4).

Univariate and multivariate regression analysis revealed a greater than $30 \%$ risk reduction for severe vasospasm for each step of mobilization achieved during the first 4 days after aneurysm repair (Table 5). Hunt and Hess grade was not a predictor of severe vasospasm in the multiple regression analysis, whereas older age reduced the risk and large amounts of blood in the subarachnoid space (high Fisher grade) increased the risk of severe vasospasm. A corresponding analysis with clinical vasospasm as the dependent variable produced similar results other than age not being a significant predictor. One patient in the early rehab group developed neurological deterioration 12 days
TABLE 2. Entrance variables in the control and early rehab groups

\begin{tabular}{|c|c|c|}
\hline Variable & Control, $n=77$ & $\begin{array}{l}\text { Early Rehab, } \\
\qquad \mathrm{n}=94\end{array}$ \\
\hline \multicolumn{3}{|l|}{ Patient demographic data } \\
\hline Age in yrs, median (range) & $54(25-79)$ & $57(25-81)$ \\
\hline Sex, F/M (\%) & $64: 36$ & $70: 30$ \\
\hline Weight in kg, median (range) & $75(42-149)$ & $70(43-125)$ \\
\hline Active smoking, $\%$ & 58 & 58 \\
\hline \multicolumn{3}{|l|}{ Comorbidity, \% } \\
\hline Musculoskeletal diseases & 17 & 27 \\
\hline Chronic pulmonary disease & 8 & 10 \\
\hline $\begin{array}{l}\text { Cardiac \& peripheral arterial } \\
\text { vascular disorders }\end{array}$ & 4 & 6 \\
\hline Arterial hypertension & 18 & 20 \\
\hline Arrhythmia & 1 & 6 \\
\hline History of psychosis & 7 & 2 \\
\hline Depression & 12 & 11 \\
\hline Stroke w/o sequelae & 5 & 3 \\
\hline Hypothyroidism & 7 & 7 \\
\hline Diabetes mellitus & 8 & 3 \\
\hline Multiple ( $\geq 2)$ comorbidities & 26 & 29 \\
\hline \multicolumn{3}{|l|}{ SAH characteristics } \\
\hline \multicolumn{3}{|l|}{$\begin{array}{l}\text { Hunt and Hess grade prior to } \\
\text { aneurysm repair, } \%^{*}\end{array}$} \\
\hline I & 27 & 22 \\
\hline II & 37 & 37 \\
\hline III & 14 & 23 \\
\hline IV & 17 & 11 \\
\hline V & 5 & 7 \\
\hline $\begin{array}{l}\text { GCS score prior to aneurysm } \\
\text { repair, mean } \pm \text { SD } \dagger\end{array}$ & $12.7 \pm 3.4$ & $12.5 \pm 3.6$ \\
\hline $\begin{array}{l}\text { Rebleeding prior to aneurysm } \\
\text { repair, \% }\end{array}$ & 7 & 9 \\
\hline LeRoux score $\geq 5, \% \ddagger$ & 18 & 23 \\
\hline $\begin{array}{l}\text { Modified Fisher grade: thick SAH, } \\
\% \S\end{array}$ & 49 & 62 \\
\hline \multicolumn{3}{|l|}{ Aneurysm location, \% } \\
\hline Frontal & 52 & 45 \\
\hline Middle & 34 & 41 \\
\hline Posterior & 14 & 14 \\
\hline \multicolumn{3}{|l|}{ Medication prior to ictus } \\
\hline Acetylsalicylic acid, no. (\%) & $9(12)$ & $8(8)$ \\
\hline Warfarin, no. (\%) & $1(1)$ & $4(4)$ \\
\hline
\end{tabular}

Frontal = anterior cerebral arteries; GCS = Glasgow Coma Scale; middle = middle cerebral arteries and internal carotid arteries; posterior = vertebrobasilar arteries.

* Hunt and Hess.

† Teasdale and Jennett.

$\ddagger$ LeRoux et al.

$\S$ Kramer et al. 
TABLE 3. Treatment variables in the control and early rehab groups

\begin{tabular}{|c|c|c|}
\hline Variable & $\begin{array}{l}\text { Control, } \\
n=77\end{array}$ & $\begin{array}{l}\text { Early Rehab, } \\
\qquad \mathrm{n}=94\end{array}$ \\
\hline $\begin{array}{l}\text { Days from ictus to aneurysm } \\
\text { repair, median (range) }\end{array}$ & $0.43(0-6)$ & $0.36(0-8)$ \\
\hline Surgical aneurysm repair, \% & 51 & 47 \\
\hline Pts w/ tracheostomy, \% & 25 & 32 \\
\hline External ventricular drain, \% & 57 & 65 \\
\hline Lumbar drain, \% & 48 & 45 \\
\hline $\begin{array}{l}\text { Total ml of CSF drained, median } \\
\text { (range) }\end{array}$ & $2486(20-6512)$ & $2156(5-8026)$ \\
\hline Ventriculoperitoneal shunt, \% & 27 & 21 \\
\hline w/in 90 days after the ictus, $\%$ & 27 & 20 \\
\hline $\begin{array}{l}\text { Days from ictus to shunt place- } \\
\text { ment, median (range) }\end{array}$ & $24(14-56)$ & $32(14-130)$ \\
\hline \multicolumn{3}{|l|}{ Medication initiated after the ictus } \\
\hline $\begin{array}{l}\text { Heparin, mean IU/day } \\
\text { (range) } \dagger\end{array}$ & $405(0-13,017)$ & $84(0-3000)$ \\
\hline $\begin{array}{l}\text { Antiplatelet drugs, mean mg/ } \\
\text { day (range) }\end{array}$ & $19(0-117)$ & $15(0-123)$ \\
\hline $\begin{array}{l}\text { Dalteparin, mean IU/day } \\
\text { (range) }\end{array}$ & 2995 (547-9259) & $3119(0-10,667)$ \\
\hline $\begin{array}{l}\text { Antibiotics, mean no. of days } \\
\text { used (range) }\end{array}$ & $8.9(0-48)$ & $8.4(0-35)$ \\
\hline $\begin{array}{l}\text { Periop use of corticoste- } \\
\text { roids, \% } \%\end{array}$ & 30 & 13 \\
\hline $\begin{array}{l}\text { Intravenous nimodipine, } \\
\text { median no. of days used } \\
\text { (range) }\end{array}$ & $7(0-31)$ & $9(0-25)$ \\
\hline $\begin{array}{l}\text { Oral nimodipine, median } \\
\text { no. of days used until dis- } \\
\text { charge (range) }\end{array}$ & $3(0-21)$ & $2(0-23)$ \\
\hline \multicolumn{3}{|l|}{$\begin{array}{l}\text { IU }=\text { international units; } \text { pts }=\text { patients. } \\
{ }^{*} p=0.07 \text {. } \\
\dagger p=0.061 \\
\ddagger p<0.01\end{array}$} \\
\hline
\end{tabular}

after the ictus, shortly after he was mobilized to standing at bedside. A digital subtraction angiography study performed under general anesthesia revealed no severe vasospasm. Nevertheless, he received intraarterial nimodipine. An MRI study revealed infarction in the left distal middle cerebral artery territory.

\section{Other Complications}

Length of stay, frequency of unintended removal of lines/tubes (none during mobilization), and clinical status at discharge were similar in both groups. Table 6 presents the frequency of other complications within 90 days after the ictus, showing similar complication rates in the 2 groups. Having previously suffered multiple episodes of DVT due to homozygotic Leiden mutation and being on warfarin prior to aSAH, 1 patient from the early rehab group was excluded from the analysis because of thromboembolic complications.

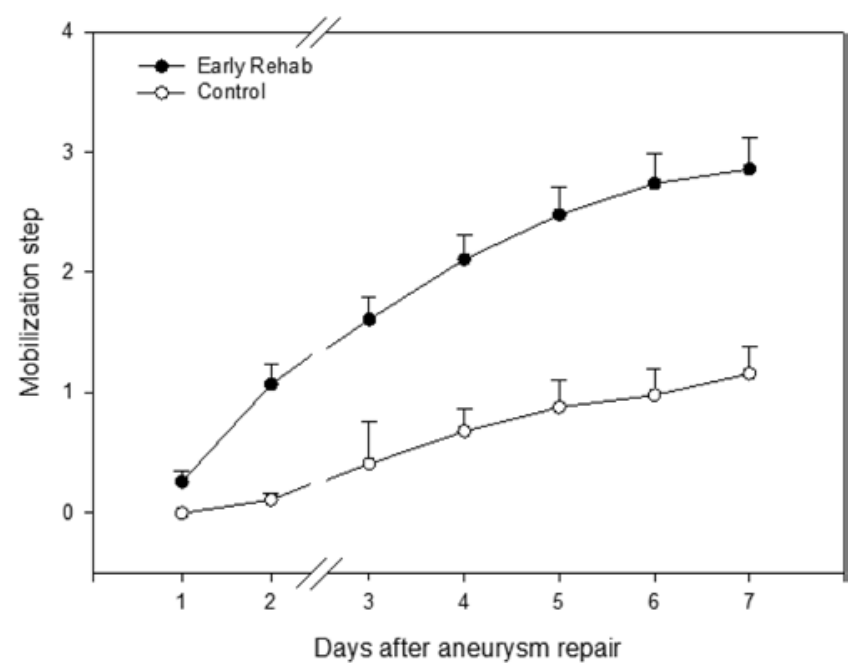

FIG. 2. Graph illustrating the much higher average mobilization step in the early rehab group (black circles) compared with the control group (white circles) versus days after aneurysm repair. The broken line on the $x$-axis between Days 2 and 3 indicates that the number of patients was successively reduced due to discharge. The standard error of the mean is given for each data point.

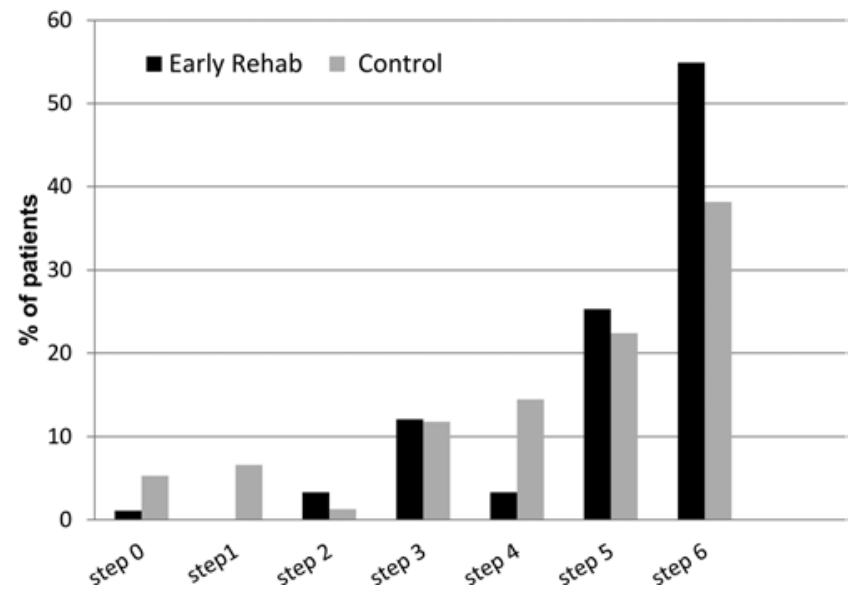

FIG. 3. Bar graph visualizing the higher average mobilization level at discharge in the early rehab group (black) compared with the control group (gray).

One patient in the early rehab group died before discharge. This patient had polycystic kidney disease and chronic renal failure as well as chronic obstructive lung disease prior to ictus and developed multiple organ failure.

\section{Discussion}

The core finding of the present study was that early mobilization and rehabilitation after aSAH is feasible and safe. We found less clinical vasospasm in the early rehab group, and a risk reduction of greater than $30 \%$ for severe vasospasm for each step of mobilization achieved during the first 4 days after aneurysm repair. Despite early mobilization, we saw no reduction in complications including respiratory tract/pulmonary infections, thromboembolic events, and death. Evaluation of the long-term effects 
TABLE 4. Percentage of patients with cerebral vasospasm in the control and early rehab groups

\begin{tabular}{|c|c|c|}
\hline Variable & $\begin{array}{c}\% \text { Control, } \\
n=72\end{array}$ & $\begin{array}{c}\% \text { Early Rehab, } \\
n=84\end{array}$ \\
\hline \multicolumn{3}{|l|}{ Radiological vasospasm } \\
\hline None & 35 & 41 \\
\hline Moderate (<50\% narrowing), $\geq 1$ vessel & 46 & 43 \\
\hline Severe ( $\geq 50 \%$ narrowing), $\geq 1$ vessel & 19 & 16 \\
\hline \multicolumn{3}{|l|}{ Ultrasonic vasospasm } \\
\hline None & 34 & 48 \\
\hline $\begin{array}{l}\text { Moderate (hemispheric ratio } 3.5-<6 \text { ), } \\
\geq 1 \text { vessel }^{*}\end{array}$ & 33 & 35 \\
\hline $\begin{array}{l}\text { Severe } \text { (hemispheric ratio } \geq 6 \text { or } \\
\quad \text { velocity }>200 \mathrm{~cm} / \mathrm{sec} \text { ), } \geq 1 \text { vessel }\end{array}$ & 33 & 17 \\
\hline Clinical vasospasm† & 29 & 14 \\
\hline Pts treated w/intraarterial nimodipine & 4 & 10 \\
\hline
\end{tabular}

of early mobilization and rehabilitation was beyond the scope of this study.

\section{Early Mobilization}

Earlier and quicker progression in mobilization in the early rehab group was performed without increased rates of neurosurgical and medical complications. It is hence feasible and safe to mobilize patients with aSAH as soon as the first day after aneurysm repair. Recommended mobilization levels at various time points after aneurysm repair have rarely been described. Blissitt et al. ${ }^{7}$ deemed head elevation up to $45^{\circ}$ to be safe in patients with aSAH. The effect of head elevation in excess of $45^{\circ}$ and mobilization of patients out of bed is not yet known. However, our data support that it is safe if individual clinical thresholds of surveillance parameters are respected and if there are no signs of patient discomfort.

\section{Effect of Early Mobilization and Rehabilitation on Cerebral Vasospasm}

The diagnosis of clinical vasospasm is difficult because it is based on exclusion of other causes of delayed neurological deterioration. ${ }^{21}$ This uncertainty, however, should have introduced a similar bias in both of our patient groups. We may assume that our mobilization protocol made the nurses and doctors at the NIW quite alert with regard to clinical vasospasm, possibly leading to an overrating of clinical vasospasm in the early rehab group. Our findings, however, were the opposite; we found less clinical vasospasm in the early rehab group. The frequency of clinical vasospasm in patients in our control group corresponded to the findings of Awad et al., ${ }^{5}$ who treated patients according to a protocol similar to our standard protocol. The course of vasospasm did not differ between groups, except that vasospasm resolved somewhat more quickly in the early rehab group. The course of vasospasm in patients in our control group is similar to the time frame described in other studies. ${ }^{29}$

Despite extensive research, the exact pathophysiological mechanism behind the development of cerebral vasospasm has still not been delineated. There seems, however, to be some consensus that cerebral vasospasm represents an immunological/inflammatory response linked to the amount of blood in the subarachnoid space. ${ }^{14}$ Our regres-
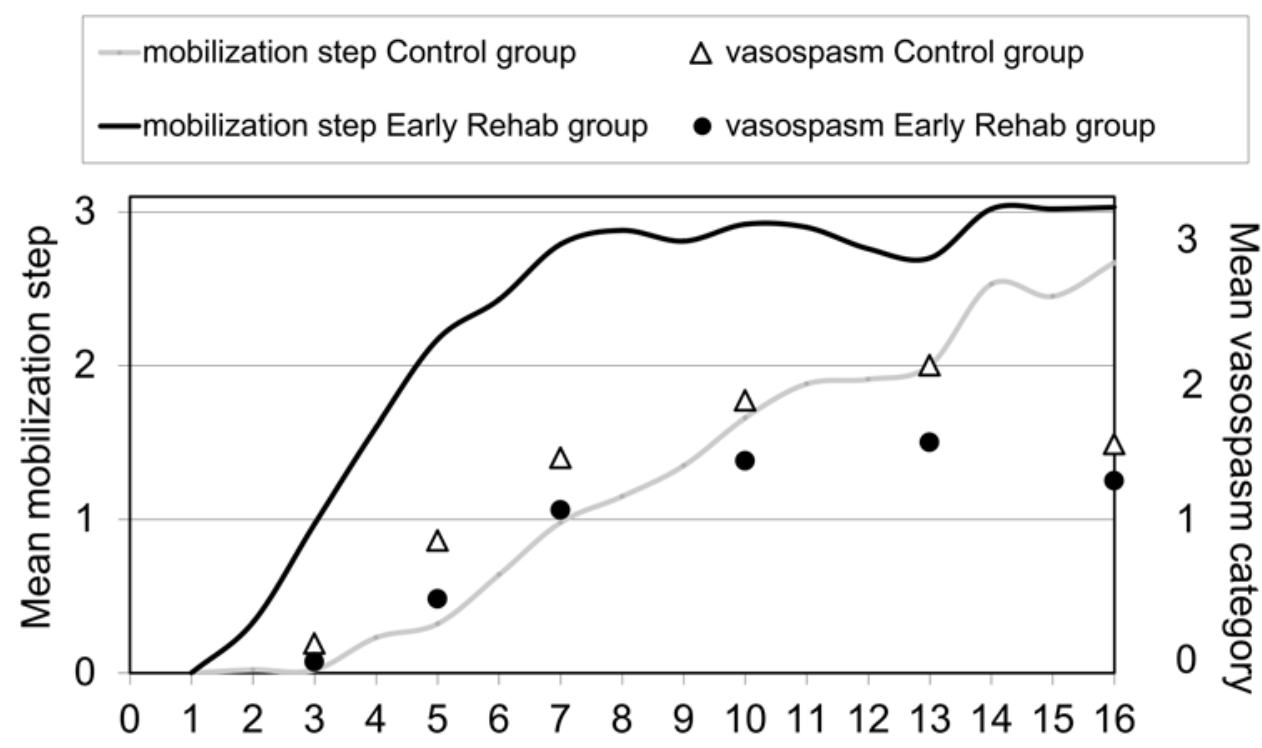

Days after ictus

FIG. 4. Graph showing the mean highest grade of vasospasm documented with either method versus the mean mobilization step. The mobilization step was higher and vasospasm tended to be less frequent in the early rehab group. The y axis shows the mean mobilization step for each day after the ictus and the mean vasospasm category (highest category scored within time frame): $0=$ no vasospasm; $1=$ moderate vasospasm in 1 vessel; $2=$ moderate vasospasm in several vessels; 3 = severe vasospasm in 1 vessel; and 4 = severe vasospasm in several vessels. 
TABLE 5. Univariate and multivariate logistic regression with severe vasospasm as the dependent variable

\begin{tabular}{|c|c|c|c|c|c|c|}
\hline \multirow[b]{2}{*}{ Variable } & \multicolumn{3}{|c|}{$\begin{array}{l}\text { Univariate } \\
\text { Analysis }\end{array}$} & \multicolumn{3}{|c|}{$\begin{array}{l}\text { Multivariate Forward/Backward } \\
\text { Stepwise Logistic Regression }\end{array}$} \\
\hline & OR & $95 \% \mathrm{Cl}$ & Significance & OR & $95 \% \mathrm{Cl}$ & Significance \\
\hline Clinical Hunt \& Hess grade* & 1.425 & $1.040-1.953$ & 0.030 & & & NS \\
\hline Modified Fisher grade $†$ & 1.630 & $1.124-2.363$ & 0.010 & 1.655 & $1.080-2.534$ & 0.021 \\
\hline Intraventricular blood (LeRoux score) $\ddagger$ & 1.101 & $0.991-1.225$ & NS $(0.074)$ & & & NS \\
\hline Max mobilization step w/in first 4 days after aneurysm repair & 0.640 & $0.475-0.863$ & 0.003 & 0.686 & $0.497-0.946$ & 0.021 \\
\hline Age & 0.966 & $0.933-1.001$ & 0.056 & 0.949 & $0.911-0.989$ & 0.012 \\
\hline If surgical aneurysm repair & 0.599 & $0.271-1.321$ & NS & & & NS \\
\hline $\begin{array}{l}\text { Max = maximum; NS = not significant. } \\
\text { * Hunt and Hess. } \\
\dagger \text { Kramer et al. } \\
\ddagger \text { LeRoux et al. }\end{array}$ & & & & & & \\
\hline
\end{tabular}

sion analysis hence delineated the amount of subarachnoid blood to be a predictor of severe as well as clinical vasospasm. The same analysis showed that older age was slightly protective with regard to developing severe vasospasm; this finding corroborates with the study of Inagawa, ${ }^{17}$ but not of Lanzino et al. ${ }^{25}$

Our strongest predictor of severe and clinical vasospasm, however, was the degree of mobilization reached within the first 4 days after aneurysm repair. Each step of mobilization reduced the risk of vasospasm by $30 \%$. This is in consensus with the retrospective and laboratory study of Riordan et al., ${ }^{32}$ who found that mild exercise before the 4th day after aneurysm repair reduced symptomatic vasospasm from $55.2 \%$ to $13.6 \%$ without promoting neuronal injury. Frequent, systematic mobilization represents a form of kinetic treatment parallel to the "head-shaking method" 30,39 that was meant to help dissolve residual blood clots. The method is often used in conjunction with cisternal and ventricular lavage, ${ }^{15}$ and there is strong evidence that it leads to a reduction in vasospasm and improves outcome. ${ }^{15,22,30,39}$ Kawamoto et al. ${ }^{22}$ even concluded that "the effect of head shaking in preventing vasospasm is the most definite compared with any existing treatment protocol."

In our department, we aggressively drain CSF. In patients who frequently change body posture, there is less sedimentation of blood products, which are then likely to be drained with the CSF. CSF frequently becomes tinged with blood after a patient has been moved (e.g., from the bed to the CT table). It is possible that more blood products were drained from patients in the early rehab group and they hence developed less vasospasm. In parallel with the head-shaking method, this effect was most prominent for clinical vasospasm and less so for angiographically documented vasospasm. ${ }^{22}$ Unlike the head-shaking method, which may cause harm in up to $30 \%$ of patients ${ }^{4}$ (including new cerebral hematoma and brain edema), early mobilization represents a mild kinetic therapy that is effective but safe.

The increased use of intraarterial nimodipine in patients in our early rehab group as treatment for severe/clinical vasospasm probably reflects our larger focus on the problem of vasospasm during the year 2012. This more liberal attitude is in accordance with a study that has shown intraarterial nimodipine to be an effective and safe interven- tion in the management of severe vasospasm refractory to hyperdynamic treatment. ${ }^{29}$ Intraarterial nimodipine may, however, have contributed to stroke development in the patient in the early rehab group whose health deteriorated, because this case was very similar to the case described by Ryu et al. ${ }^{33}$

\section{Effect of Early Mobilization and Rehabilitation on Hydrocephalus}

The degree of acute hydrocephalus is largely determined by the amount of subarachnoid blood. Also, patients in a poorer clinical condition more often need CSF drainage. ${ }^{12}$ The amount of subarachnoid and ventricular blood,

TABLE 6. Frequency of complications within 90 days after aneurysm repair in the control and early rehab groups and status at discharge

\begin{tabular}{|c|c|c|}
\hline Complication & $\begin{array}{c}\text { Control, } \\
\mathrm{n}=77\end{array}$ & $\begin{array}{c}\text { Early Rehab, } \\
\quad n=94\end{array}$ \\
\hline $\begin{array}{l}\text { Cerebral infarction acquired after the } \\
\text { ictus, } \%\end{array}$ & 40 & 29 \\
\hline Respiratory tract/pulmonary infection, $\%$ & 58 & 54 \\
\hline DVT, no. of cases* & 2 & 2 \\
\hline PE, no. of cases $\dagger$ & 1 & 2 \\
\hline Rebleeding after aneurysm repair & 0 & 0 \\
\hline $\begin{array}{l}\text { Highest measured body temperature in } \\
{ }^{\circ} \mathrm{C} \text {, median (range) }\end{array}$ & $38.5(37-40)$ & $38.7(37-40)$ \\
\hline $\begin{array}{l}\text { No. of days body temperature }>37^{\circ} \mathrm{C} \text {, } \\
\text { median (range) }\end{array}$ & $8(1-56)$ & $10(0-35)$ \\
\hline Death before discharge, no. of pts & 0 & 1 \\
\hline Death w/in 90 days, \% & 5.2 & 5.3 \\
\hline GCS score at discharge, mean \pm SD $\S$ & $14.1 \pm 1.5$ & $13.9 \pm 1.9$ \\
\hline Length of stay in days, median (range) & $14.5(2-61)$ & $13.9(3-37)$ \\
\hline \multicolumn{3}{|c|}{$\begin{array}{l}\text { * Time to DVT in each patient: } 11 \text { and } 60 \text { days (control group); } 15 \text { and } 49 \text { days } \\
\text { (early rehab group). } \\
\text { † Time to PE in each patient: } 14 \text { days (control group); } 8 \text { and } 32 \text { days (early } \\
\text { rehab group). } \\
\ddagger \text { Time to mortality in each patient: on Days } 36,42,46 \text {, and } 60 \text { (control group); } \\
\text { on Days } 16,27,29,36 \text {, and } 66 \text { (early rehab group). } \\
\S \text { Teasdale and Jennett. }\end{array}$} \\
\hline
\end{tabular}


as well as the clinical condition of patients, was similar in both of our groups. Thus, our finding that the total amount of CSF drained and the frequency of shunt placement were similar in the 2 groups is not surprising. The need for implantation of a CSF shunt for chronic hydrocephalus varies with patient age, amount and localization of blood, clinical condition of the patient, amount of CSF drained during the acute stage, and institutional protocols. ${ }^{12}$ Among patients in the early rehab group, the time between ictus and shunt insertion tended to be longer than in the control group. It is possible that increased blood lavage in the early rehab group contributed to this effect.

\section{Effect of Early Rehabilitation on Other Complications}

New cerebral infarctions tended to occur less frequently in the early rehab group, an effect that was also described in head-shaking studies..$^{15,22,30}$ There was a trend for more patients with antecedent cardiac arrhythmia in the early rehab group, which could have made them more prone to arterial thromboembolic complications. The clinical relevance of this finding is uncertain. Moreover, heparin tended to be used more often in the control group and could have had a protective effect regarding thromboembolism. Given a positive effect, ${ }^{1}$ the more frequent use of corticosteroids in the control group could have masked a beneficial effect of early rehabilitation.

Pulmonary physiotherapy is standard treatment for prevention of respiratory tract/pulmonary infections in our department; i.e., it was applied to the same degree in both groups. Therefore, one would not expect an auxiliary effect from early mobilization and rehabilitation.

Patients were not screened for DVT and PE in our study, but they underwent diagnostic tests upon clinical suspicion. We therefore cannot compare our incidence rates with those in the study by Serrone et al., ${ }^{34}$ who reported incidences of DVT and PE of $9.7 \%$ and $2 \%$, respectively. Our numbers compare better with those of Kim and Brophy, ${ }^{23}$ who found that the incidence of clinical venous thromboembolism was $6.7 \%$. The lower incidence of DVT and PE in our study, as well as the low mortality rate, could be explained by the exclusion of the sickest patients from the study (i.e., those who remained in our ICU for the entire stay).

\section{Limitations and Strengths of the Study}

Several limitations should be considered when interpreting the results of this study. First, patients who were managed in the ICU for their entire stay at our hospital were excluded. These patients were those in the poorest clinical condition and who were most susceptible to experiencing complications. Exclusion of these patients probably contributed to the low frequency of complications in both study groups and made it more difficult to detect potential positive effects of our intervention. Future studies should not only confirm our findings but also include patients on invasive mechanical respiratory support. Second, the sample size of the study limited the number of covariates that could be controlled for, especially regarding complications with low incidence such as thromboembolic events. Third, a historical cohort cannot eliminate the possibility of confounding bias by unmeasured confounders.

A strength of the present study was that patient demo- graphic data and aSAH characteristics were very similar in the 2 groups and hence well suited to examine the effect of early rehabilitation. Both groups were treated in accordance with the same strict institutional guidelines by the same neurosurgeons, anesthesiologists, and nurses. This created standardized study conditions and hence reduced bias that, for instance, would be present when comparing the results of different neurosurgical departments. The ideal study setting would have been a randomized controlled trial within the same department. However, it would be impossible to blind the hospital staff to group affiliations of patients. Furthermore, we would actually increase bias due to a contamination effect of the intervention spreading to the control group. Despite its pitfalls, we believe that our study design was the best obtainable. Nevertheless, to our knowledge this study is one of only a few, and is so far the largest prospective study to investigate early mobilization and rehabilitation in patients with aSAH.

\section{Conclusions}

Early mobilization and rehabilitation after aSAH is safe and feasible. Early rehabilitation leads to an earlier and higher degree of mobilization without increasing neurosurgical complications. Cerebral vasospasm is alleviated by early rehabilitation with each step of mobilization achieved during the first 4 days after aneurysm repair, reducing the risk for severe and clinical vasospasm by $30 \%$.

\section{References}

1. Adamczyk P, He S, Amar AP, Mack WJ: Medical management of cerebral vasospasm following aneurysmal subarachnoid hemorrhage: a review of current and emerging therapeutic interventions. Neurol Res Int 2013:462491, 2013

2. Albano C, Comandante L, Nolan S: Innovations in the management of cerebral injury. Crit Care Nurs Q 28:135-149, 2005

3. Andelic N, Bautz-Holter E, Ronning P, Olafsen K, Sigurdardottir S, Schanke AK, et al: Does an early onset and continuous chain of rehabilitation improve the long-term functional outcome of patients with severe traumatic brain injury? J Neurotrauma 29:66-74, 2012

4. Aoki N: "Head-shaking syndrome" neurological deterioration during continuous head-shaking as an adjunct to cisternal irrigation for clot removal in patients with acute subarachnoid haemorrhage. Acta Neurochir (Wien) 132:20-25, 1995

5. Awad IA, Carter LP, Spetzler RF, Medina M, Williams FC Jr: Clinical vasospasm after subarachnoid hemorrhage: response to hypervolemic hemodilution and arterial hypertension. Stroke 18:365-372, 1987

6. Bederson JB, Connolly ES Jr, Batjer HH, Dacey RG, Dion JE, Diringer MN, et al: Guidelines for the management of aneurysmal subarachnoid hemorrhage: a statement for healthcare professionals from a special writing group of the Stroke Council, American Heart Association. Stroke 40:994-1025, 2009

7. Blissitt PA, Mitchell PH, Newell DW, Woods SL, Belza B: Cerebrovascular dynamics with head-of-bed elevation in patients with mild or moderate vasospasm after aneurysmal subarachnoid hemorrhage. Am J Crit Care 15:206-216, 2006

8. Bode RK, Heinemann AW, Semik P: Measurement properties of the Galveston Orientation and Amnesia Test (GOAT) and improvement patterns during inpatient rehabilitation. J Head Trauma Rehabil 15:637-655, 2000

9. Cifu DX, Stewart DG: Factors affecting functional outcome after stroke: a critical review of rehabilitation interventions. Arch Phys Med Rehabil 80 (5 Suppl 1):S35-S39, 1999 
10. Diserens K, Michel P, Bogousslavsky J: Early mobilisation after stroke: Review of the literature. Cerebrovasc Dis 22:183-190, 2006

11. Diserens K, Moreira T, Hirt L, Faouzi M, Grujic J, Bieler G, et al: Early mobilization out of bed after ischaemic stroke reduces severe complications but not cerebral blood flow: a randomized controlled pilot trial. Clin Rehabil 26:451-459, 2012

12. Erixon HO, Sorteberg A, Sorteberg W, Eide PK: Predictors of shunt dependency after aneurysmal subarachnoid hemorrhage: results of a single-center clinical trial. Acta Neurochir (Wien) 156:2059-2069, 2014

13. European Stroke Organisation (ESO) Executive Committee: Guidelines for management of ischaemic stroke and transient ischaemic attack 2008. Cerebrovasc Dis 25:457-507, 2008

14. Fisher CM, Kistler JP, Davis JM: Relation of cerebral vasospasm to subarachnoid hemorrhage visualized by computerized tomographic scanning. Neurosurgery 6:1-9, 1980

15. Hänggi D, Liersch J, Turowski B, Yong M, Steiger HJ: The effect of lumboventricular lavage and simultaneous lowfrequency head-motion therapy after severe subarachnoid hemorrhage: results of a single center prospective Phase II trial. J Neurosurg 108:1192-1199, 2008

16. Hunt WE, Hess RM: Surgical risk as related to time of intervention in the repair of intracranial aneurysms. J Neurosurg 28:14-20, 1068, 1968

17. Inagawa $\mathrm{T}$ : Cerebral vasospasm in elderly patients treated by early operation for ruptured intracranial aneurysms. Acta Neurochir (Wien) 115:79-85, 1992

18. Indredavik B, Bakke F, Slordahl SA, Rokseth R, Hâheim LL: Treatment in a combined acute and rehabilitation stroke unit: which aspects are most important? Stroke 30:917-923, 1999

19. Jessup RL: Interdisciplinary versus multidisciplinary care teams: do we understand the difference? Aust Health Rev 31:330-331, 2007

20. Karic T, Sorteberg A, Haug Nordenmark T, Becker F, Roe C Early rehabilitation in patients with acute aneurysmal subarachnoid hemorrhage. Disabil Rehabil 37:1446-1454, 2015

21. Kassell NF, Boarini DJ, Adams HP Jr, Sahs AL, Graf CJ, Torner JC, et al: Overall management of ruptured aneurysm: comparison of early and late operation. Neurosurgery 9:120-128, 1981

22. Kawamoto S, Tsutsumi K, Yoshikawa G, Shinozaki MH, Yako K, Nagata K, et al: Effectiveness of the head-shaking method combined with cisternal irrigation with urokinase in preventing cerebral vasospasm after subarachnoid hemorrhage. J Neurosurg 100:236-243, 200

23. Kim KS, Brophy GM: Symptomatic venous thromboembolism: incidence and risk factors in patients with spontaneous or traumatic intracranial hemorrhage. Neurocrit Care 11:28-33, 2009

24. Kramer AH, Hehir M, Nathan B, Gress D, Dumont AS, Kassell NF, et al: A comparison of 3 radiographic scales for the prediction of delayed ischemia and prognosis following subarachnoid hemorrhage. J Neurosurg 109:199-207, 2008

25. Lanzino G, Kassell NF, Germanson TP, Kongable GL, Truskowski LL, Torner JC, et al: Age and outcome after aneurysmal subarachnoid hemorrhage: why do older patients fare worse? J Neurosurg 85:410-418, 1996

26. LeRoux PD, Haglund MM, Newell DW, Grady MS, Winn HR: Intraventricular hemorrhage in blunt head trauma: an analysis of 43 cases. Neurosurgery 31:678-685, 1992

27. Lindegaard KF, Nornes H, Bakke SJ, Sorteberg W, Nakstad $P$ : Cerebral vasospasm after subarachnoid haemorrhage investigated by means of transcranial Doppler ultrasound. Acta Neurochir Suppl 42:81-84, 1988

28. Mendez-Tellez PA, Nusr R, Feldman D, Needham DM: Early physical rehabilitation in the ICU: a review for the neurohospitalist. Neurohospitalist 2:96-105, 2012

29. Musahl C, Henkes H, Vajda Z, Coburger J, Hopf N: Continu- ous local intra-arterial nimodipine administration in severe symptomatic vasospasm after subarachnoid hemorrhage. Neurosurgery 68:1541-1547, 2011

30. Nakagomi T, Furuya K, Nagashima H, Tanaka J, Ishii T, Takanashi S, et al: Surgical procedure and results of cisternal washing therapy for the prevention of cerebral vasospasm following SAH. Acta Neurochir Suppl 110:105-109, 2011

31. Olkowski BF, Devine MA, Slotnick LE, Veznedaroglu E, Liebman KM, Arcaro ML, et al: Safety and feasibility of an early mobilization program for patients with aneurysmal subarachnoid hemorrhage. Phys Ther 93:208-215, 2013

32. Riordan MA, Kyle M, Dedeo C, Villwock MR, Bauer M, Vallano ML, et al: Mild exercise reduces cerebral vasospasm after aneurysm subarachnoid hemorrhage: a retrospective clinical study and correlation with laboratory investigation. Acta Neurochir Suppl 120:55-61, 2015

33. Ryu CW, Koh JS, Yu SY, Kim EJ: Vasogenic edema of the basal ganglia after intra-arterial administration of nimodipine for treatment of vasospasm. J Korean Neurosurg Soc 49:112-115, 2011

34. Serrone JC, Wash EM, Hartings JA, Andaluz N, Zuccarello M: Venous thromboembolism in subarachnoid hemorrhage. World Neurosurg 80:859-863, 2013

35. Sörbo A, Rydenhag B, Sunnerhagen KS, Blomqvist M, Svensson S, Emanuelson I: Outcome after severe brain damage, what makes the difference? Brain Inj 19:493-503, 2005

36. Sorteberg W, Slettebø H, Eide PK, Stubhaug A, Sorteberg A: Surgical treatment of aneurysmal subarachnoid haemorrhage in the presence of 24-h endovascular availability: management and results. Br J Neurosurg 22:53-62, 2008

37. Steiner T, Juvela S, Unterberg A, Jung C, Forsting M, Rinkel G: European Stroke Organization guidelines for the management of intracranial aneurysms and subarachnoid haemorrhage. Cerebrovasc Dis 35:93-112, 2013

38. Stern M, Chang D, Odell M, Sperber K: Rehabilitation implications of non-traumatic subarachnoid haemorrhage. Brain Inj 20:679-685, 2006

39. Suzuki IS, Takahashi H: Effect of head-shaking method on clot removal in cisternal irrigation, in Sano K (ed): Cerebral Vasospasm. Tokyo: University of Tokyo Press, 1990, pp 314-316

40. Teasdale G, Jennett B: Assessment of coma and impaired consciousness. A practical scale. Lancet 2:81-84, 1974

41. van Gijn J, Kerr RS, Rinkel GJ: Subarachnoid haemorrhage. Lancet 369:306-318, 2007

\section{Disclosures}

The authors report no conflict of interest concerning the materials or methods used in this study or the findings specified in this paper.

\section{Author Contributions}

Conception and design: Sorteberg, Karic, Røe, Nordenmark, Becker. Acquisition of data: Sorteberg, Karic, Nordenmark. Analysis and interpretation of data: all authors. Drafting the article: Sorteberg, Karic, Røe, Nordenmark, Sorteberg. Critically revising the article: Karic, Røe, Nordenmark, Becker, Sorteberg. Reviewed submitted version of manuscript: all authors. Approved the final version of the manuscript on behalf of all authors: Sorteberg. Statistical analysis: Sorteberg, Karic, Røe. Administrative/ technical/material support: Sorteberg, Røe. Study supervision: Sorteberg, Røe, Nordenmark, Becker, Sorteberg.

\section{Correspondence}

Angelika Sorteberg, Department of Neurosurgery, Oslo University Hospital-Rikshospitalet, Sognvannsvn 20, PB 4950 Nydalen, Oslo 0424, Norway. email: angelika.sorteberg@ous-hf.no. 